\title{
METHOdS OF COMMAND RECOGNITION USING SINGLE-CHANNEL EEGS
}

\author{
Wei-Ho Tsai, Cin-Hao Ma, and Varinya Phanichraksaphong \\ Department of Electronic Engineering, National Taipei University of Technology, \\ Taiwan, ROC
}

\begin{abstract}
This work proposes to recognize a user's commands by analysing his/her brainwaves captured with single channel electroencephalogram (EEG). Whenever a user intends to issue one of the pre-defined commands, the proposed system prompts him/her all the candidate commands in turn. Then, the user is asked to be concentrated as possible as he/she can, when the desired command is shown. It is assumed that the concentration will present a certain pattern of "Yes" in the captured EEG, as opposed to a certain pattern of "No" when the user is relaxed. Accordingly, the task is to determine that the captured EEG is "Yes" or not. This work compares three recognition methods, respectively, based on Gaussian mixture models, hidden Markov models and recurrent neural network, and conducts experiments using 2400 test EEG samples recorded from 10 subjects.
\end{abstract}

\section{KEYWORDS}

Brainwave, Electroencephalogram, Hidden Markov Model, Recognition, Recurrent Neural Network

\section{INTRODUCTION}

Nowadays computers have so much involved with our daily lives. This development is not only mainly caused by the rapid improvements in computing efficiency, but also by the successful designs of human-computer interfaces. At first, computers using a keyboard as the interface to key-in instructions were mostly used in laboratories or factories. Later on, computers using a mouse as the operating interface spread to offices and houses. Now, tablet computers and smart phones that use a touch screen as the operating interface make computers omnipresent. As people tend to rely on computers more and more, it obviously becomes increasingly important to develop more user-friendly human-computer interfaces. The demand of user friendliness has also been a key to the development of various care technologies, such as elderly care, disabled care, and children care, which involves people not familiar with or unable to use computers [16].

However, the mainstream human-computer interfaces are mostly operated with hands. In cases when hands are occupied or not available, it becomes not very convenient for people to use these interfaces. Therefore, interfaces operated by voices, eye motions, lip motions, etc. have been the focus of R\&D. Yet, respective constraints in using these human-computer interfaces remain. For instance, in situations when people are unable or inconvenient for them to utter a voice, voice-operated interfaces will not be used. 
For computer-operating instructions originate from the brain, this research tries to measure and analyse brain intentions, further decipher the user's instructions to the computer, and finally create a brain-control interface. Nevertheless, there is no denying the severe challenges in developing a brain-control interface. Besides, equipment used to measure the brain's intentions may not be user-friendly or convenient to use. The purpose of this research is only to offer a feasibility evaluation on developing such an interface, and continual improvements based on the results in this research are expected.

Specifically, this study investigates how we can recognize a user's commands by analysing his/her brainwaves characterized with electroencephalogram (EEG). Whenever a user intends to issue one of the pre-defined commands, the proposed system prompts him/her all the candidate commands in turn. Then, the user is asked to be concentrated as possible as he/she can, when the desired command is shown. We assume that the concentration will present a certain pattern of "Yes" in the captured EEG, as opposed to a pattern of "No" when the user is relaxed. Accordingly, the task is to determine that the captured EEG is "Yes" or not.

In fact, there have been several studies discussing such kind of binary EEG classification problems. In the works [1-4], they investigate what the differences of the EEG signals are when human attempts to discriminate between target and non-target images. In the works $[5,6]$, they propose using EEG to control the direction of an electric wheelchair. The studies $[7,8]$ investigate how to extract EEG features for mental fatigue classification. The work [9] proposes a computer interface to combine gaze tracking with brainwave signals in an integrated headmounted device. Nevertheless, all of the above-mentioned studies use 32, 64, or more EEG channels in building their systems, which involve inconvenient and uncomfortable headrecording nets as well as expensive equipment, therefore unsuitable for real human-machineinterfaced applications. Recognizing this problem, this study, extending from our previous work [10], proposes using a simple, portable, and cheap instrument that extracts a single-channel EEG from the user's frontal lobe. The underlying beta waves of EEG are then distilled and used as the feature to determine the user's command. We compare three recognition methods, respectively, based on Gaussian mixture models, hidden Markov models and recurrent neural network.

The rest of this paper is organized as follows. Sec. 2 describes the concept of brainwave and EEG. Sec. 3 presents the methodology of the proposed EEG-based command recognition system. In Sec. 4, our experiment results are discussed. Then, Sec. 5 presents our conclusions and the direction of future work.

\section{BRAINWAVE AND ELECTROENCEPHALOGRAPHY}

Brainwaves can generally be divided into five categories. 1) Delta wave, with frequency ranging from 0.5 to $4 \mathrm{~Hz}$, appears mainly during deep and dreamless sleep and represents a completely unconscious state. 2) Theta wave, with frequency ranging from 4 to $8 \mathrm{~Hz}$, appears mainly during light sleep or extreme relaxation and represents a subconscious state. 3) Alpha wave, with frequency ranging from 8 to $13 \mathrm{~Hz}$, appears mainly during just awakening naturally from sleep or relaxed and represents a state of between consciousness and sub-consciousness. 4) Beta wave, with frequency ranging from 13 to $28 \mathrm{~Hz}$, appears during wide awakening, especially in thinking and studying and represents a completely conscious state. 5) Gamma wave, with frequency ranging from 28 to $50 \mathrm{~Hz}$, may appear during the formation of ideas, language and memory processing, and various types of learning. 
A method for recording the electrical activity of the human brain from the surface of the head is called Electroencephalogram (EEG). Through the use of EEG, research has found the relationship between the areas of cerebral cortex and the functions for controlling every part of a human body. For example, frontal lobe is related to planning, reasoning, movement, emotions, and parts of speech. Parietal lobe is related to recognition, orientation, and perception of stimuli. Occipital lobe is related to visual processing. Temporal lobe is related to perception and recognition of auditory stimuli.

To ensure EEG studies could be compared to each other, the International Federation of Societies for Electroencephalography and Clinical Neurophysiology developed an internationally recognized method, called the 10-20 system of electrode placement [11], which standardizes the location of scalp electrodes at specific intervals along the head in the context of an EEG experiment. As shown in Figure 1, each electrode site is given a letter to identify the lobe, along with a number to identify the hemispheric location. Specifically, "F" stands for Frontal lobe, "T" stands for Temporal lobe, "C" stands for Central lobe, "P" stands for Parietal lobe, and "O" stands for Occipital lobe.

Nowadays, a number of EEG equipment follows the 10-20 system of electrode placement. However, most of them involve inconvenient and uncomfortable head-recording nets as well as heavy cost; hence, they are unsuitable for real human-machine-interfaced applications. To deal with this problem, we seek to develop our brain-control interface by using a simple, portable, and cheap instrument.

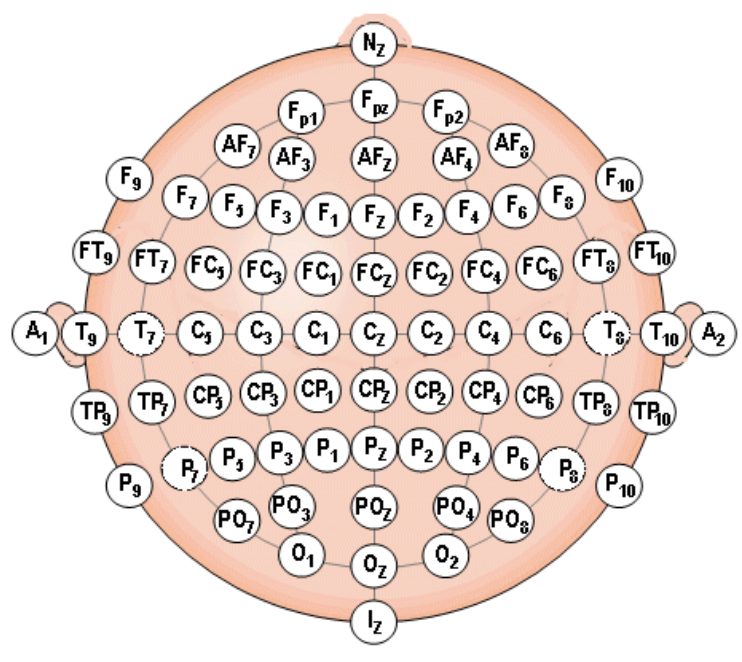

Figure 1. The 10-20 system of electrode placement (redrawn from [11]).

\section{Methodology}

The proposed framework for EEG-based command recognition is shown in Figure 2. Whenever a user intends to issue one of the pre-defined commands, the proposed system prompts him/her all the candidate commands in turn. Then, the user is asked to be concentrated as possible as he/she can, when the desired command is shown. It is assumed that the concentration will present a certain pattern of "Yes" in the captured EEG, as opposed to a certain pattern of "No" when the user is relaxed. 


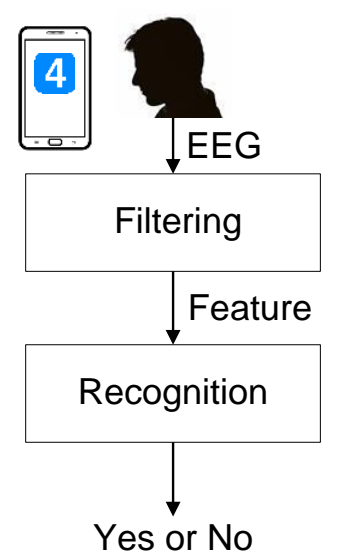

Figure. 2. The proposed command recognition system.

\subsection{EEG Measurement}

We use Mindband $\AA$ produced by NeuroSky Inc. to extract single-channel EEGs from a user's frontal lobe $\left(\mathrm{Fp}_{1}\right.$ or $\left.\mathrm{Fp}_{2}\right)$. MindBand ${ }^{\circledR}$ is a headband device that senses electrical activity via a dry-electrode. As mentioned earlier, frontal lobe is related to planning, reasoning, movement, emotions, and parts of speech; hence, EEGs extracted from frontal lobe should be able to reflect a user's command. In addition, considering beta wave is related to thinking and active concentration, we propose using beta wave extracted from the frontal lobe for command recognition.

The electrode of Mindband ${ }^{\circledR}$ headband device is placed on the forehead to capture the brainwave appearing in the area of frontal lobe. The captured signals are then transmitted to a computer via Bluetooth. Because brainwaves captured from the surface of the head are tiny, MindBand ${ }^{\circledR}$ performs amplification and filtering. After that, signals are quantized with 12-bit resolution. In addition, the power spectral density of the signal is then computed for every 0.125 sec with frequency resolution of $0.25 \mathrm{~Hz}$. Figure 3 shows an example of the captured beta wave represented by the power spectral density of the EEG. We can see from Figure 3 that beta wave in lower frequencies is somewhat different from that in higher frequencies. Hence, in general, beta wave can be further divided into two bands, $13-20 \mathrm{~Hz}$ and $21-28 \mathrm{~Hz}$, respectively, denoted by $\beta_{1}$ and $\beta_{2}$ hereafter.

\subsection{Strategy of the Command Recognition}

The recognition modular operates in two phases, training and testing. The training phase begins with EEG data collection, which acquires a large amount of EEG data labelled with "Yes" or "No". As our preliminary investigation found that the EEG pattern is diverse across different people significantly, we focus only on developing a user-dependent system, i.e., the system is trained using the pre-recorded EEG data of an individual user, and works exclusively for the user.

In the EEG training data acquisition, subjects are asked to stare the screen from which commands are prompted at random. Before that, each subject needs to choose a command in mind. If the prompted command is what the subject chose, then he/she should be concentrated 
as possible as he/she can, in order to show his/her intention. On the contrary, if the prompted command is not what the subject chose, then he/she should keep relaxed. The EEG recordings are then labelled with "Yes" or "No" for every 0.5 second.

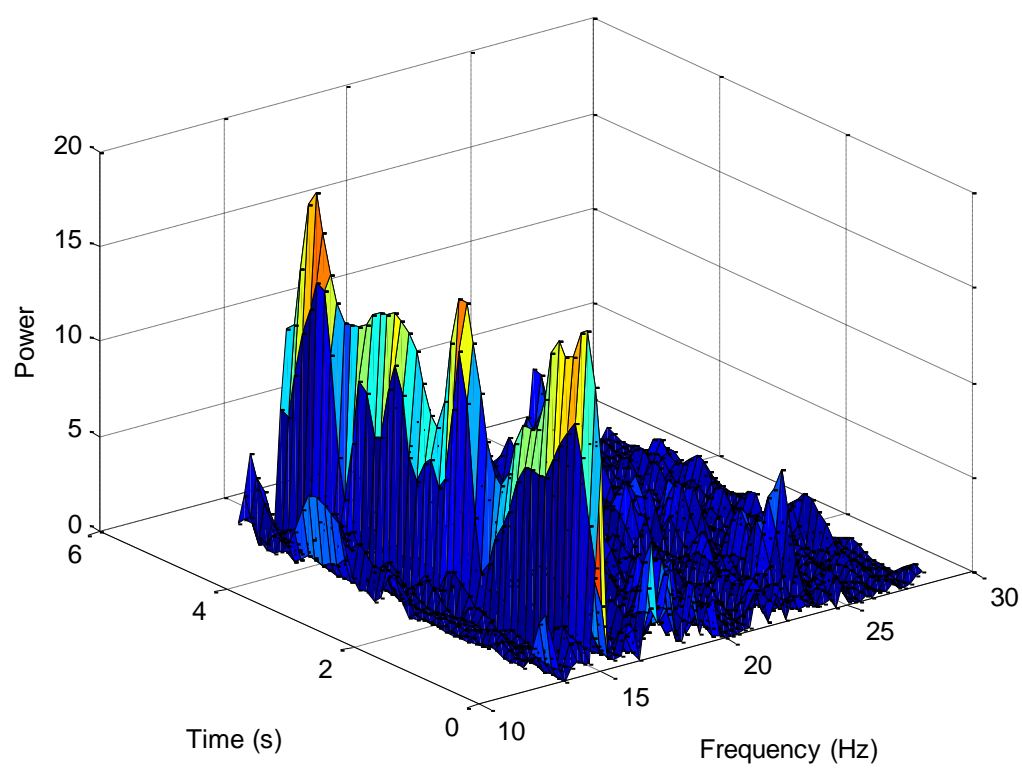

Figure. 3. An example of the captured beta wave represented by the power spectral density of the EEG.

As mentioned earlier, beta wave is related to thinking and active concentration. Hence, an intuitive approach for determining a user's command is based on the average power of the beta wave, similar to some work [12,13] using the NeuroSky's EEG device. However, our experiments found that because brainwave patterns change with time, using the average power of the beta wave for command recognition is far from stable. To deal with this problem, we propose using Gaussian mixture models, hidden Markov models or recurrent neural network to capture the dynamic information of the EEG signals.

\subsection{Command Recognition Based on Gaussian Mixture Models (GMMs)}

Gaussian Mixture Models (GMMs) are known to provide good approximations of arbitrarily shaped densities of a spectrum over a long span of time [14]. The parameters of a GMM, composed of means, covariances, and mixture weights, can be estimated using $k$-means initialization and Expectation-Maximization (EM) [15]. We create two GMMs, $\lambda_{Y}$ and $\lambda_{N}$, where the former stands for "Yes" EEG pattern, and the letter stands for "No" EEG pattern.

Let $\mathbf{O}=\left\{O_{1}, O_{2}, \ldots, O_{T}\right\}$ be the $T$-length betawave feature represented by the power spectral density of the testing EEG. The system computes the two probabilities $\operatorname{Pr}\left(\mathbf{O} \mid \lambda_{Y}\right)$ and $\operatorname{Pr}\left(\mathrm{O} \mid \lambda_{N}\right)$, e.g.,

$$
\operatorname{Pr}\left(\mathbf{O} \mid \lambda_{\mathrm{Y}}\right)=\prod_{t=1}^{T} \sum_{k=1}^{K} w_{\mathrm{Y}}^{(k)} \cdot \mathrm{N}\left(O_{t} ; \boldsymbol{\mu}_{\mathrm{Y}}^{(k)}, \mathbf{C}_{\mathrm{Y}}^{(k)}\right)
$$




$$
\mathrm{N}\left(O_{t} ; \boldsymbol{\mu}_{\mathrm{Y}}^{(k)}, \mathbf{C}_{\mathrm{Y}}^{(k)}\right)=\frac{1}{\pi^{N}\left|\mathbf{C}_{\mathrm{Y}}^{(k)}\right|} \exp \left\{-\left(O_{t}-\boldsymbol{\mu}_{\mathrm{Y}}^{(k)}\right)^{\prime} \mathbf{C}_{\mathrm{Y}}^{(k)-1}\left(O_{t}-\boldsymbol{\mu}_{\mathrm{Y}}^{(k)}\right)\right\},
$$

where $K$ is the number of mixture Gaussian components; $w_{\mathrm{Y}}^{(k)}, \boldsymbol{\mu}_{\mathrm{Y}}^{(k)}$, and $\mathbf{C}_{\mathrm{Y}}^{(k)}$ are the $k$-th mixture weight, mean, and covariance of model $\lambda_{\mathrm{Y}}$, respectively; and prime (') denotes the vector transpose. Then, a prompted command is determined as either "Yes" or "No" based on

$$
\ln \operatorname{Pr}\left(\mathbf{O} \mid \lambda_{\mathrm{Y}}\right)-\ln \operatorname{Pr}\left(\mathbf{O} \mid \lambda_{\mathrm{N}}\right) \stackrel{\text { Yes }}{\underset{\text { No }}{\leq}} \quad \theta
$$

where $\theta$ is a pre-set threshold. When considering low beta $\left(\beta_{1}\right)$ and high beta $\left(\beta_{2}\right)$ EEGs as two separate features, we can create four GMMs and modify Eq. (3) by

$$
a\left[\ln \operatorname{Pr}\left(\mathbf{O} \mid \lambda_{\mathrm{Y}, \beta_{1}}\right)-\ln \operatorname{Pr}\left(\mathbf{O} \mid \lambda_{\mathrm{N}, \beta_{1}}\right)\right]+(1-a)\left[\ln \operatorname{Pr}\left(\mathbf{O} \mid \lambda_{\mathrm{Y}, \beta_{2}}\right)-\ln \operatorname{Pr}\left(\mathbf{O} \mid \lambda_{\mathrm{N}, \beta_{2}}\right)\right] \stackrel{\text { Yes }}{>} \theta,
$$

where subscripts $\beta_{1}$ and $\beta_{2}$ denote the GMMs trained using $\beta_{1}$ and $\beta_{2}$ features, respectively, and $a$ is a weighting factor.

\subsection{Command Recognition Based on Hidden Markov Models (HMMs)}

An HMM consisting of $N$ states is completely characterized by the parameters, $\Lambda=\{\mathbf{A}, \mathbf{B}, \Pi\}$, where $\mathbf{A}=\left[a_{i j}\right]_{N \times N}$ is the probability transition matrix, representing the probability of occurring a transition from state $i$ to state $j ; \mathbf{B}=\left[b_{j}\left(O_{t}\right)\right]_{N \times 1}$ is the output probability matrix, representing the probability of observing feature $O_{t}$ at time $t$ given that the model is in state $j ; \Pi=\left[\pi_{i}\right]_{N \times 1}$ is the initial state probability vector, representing the probability that state $i$ is the initial state. HMM here functions as a random generator of the EEG signals. Basically, we create two HMMs, $\Lambda_{\mathrm{Y}}$ and $\Lambda_{\mathrm{N}}$, where the former stands for "Yes", and the letter stands for "No". However, to consider low beta $\left(\beta_{1}\right)$ and high beta $\left(\beta_{2}\right)$ EEGs as two separate features, we can create four HMMs in analogy to the procedure of the GMM-based command recognition in Sec. 3.3. During recognition, Eq. (4) is also applied to determine the prompted command as either "Yes" or "No".

\subsection{Command Recognition Based on Recurrent Neural Network}

The structure of our RNN is shown in Figure. 4. It consists of three layers of neurons, namely, input layer, which receives the beta wave feature stream, hidden layer, which models the dynamic structure and output layer, which provides the hypothesis of the user's command recognized. 
Given a stream of $K$-dimension beta wave feature vectors $\mathbf{x}[n]=\left[x_{1}[n], x_{2}[n], x_{K}[n]\right]^{\prime}$, which is the power spectral density of the EEG, the activation function of hidden neuron $j$ at frame time $n$ is defined as

$$
h_{j}[n]=\left\{1+\exp \left[-\alpha\left(\sum_{k=1}^{K} w_{k j} x_{k}[n]+\sum_{i=1}^{J} r_{i j} h_{i}[n-1]\right)\right]\right\}^{-1},
$$

where $J$ is the number of neurons in the hidden layer, $w_{k j}$ is the feed-forward connection strength from input neuron $k$ to hidden neuron $j, r_{i j}$ is the recurrent connection strength from the delayed hidden neuron $i$ to hidden neuron $j$, and $\alpha$ is a tunable parameter. The activation function of the output neuron $\square$ at time $n$ is defined as

$$
y_{\ell}[n]=\left\{1+\exp \left[-\alpha\left(\sum_{j=1}^{J} W_{j \ell} h_{j}[n]\right)\right]\right\}^{-1},
$$

where $W_{j \square}$ is the feed-forward connection strength from hidden neuron $j$ to output neuron $\square$, and $\square=1$ or 2 . The strengths $w_{k j}, r_{i j}$, and $W_{j \square}$, can be estimated using a back-propagation learning algorithm based on gradient descent minimization such that $y_{1}[n]>y_{2}[n]$ in an average sense over time $n$, when $\mathbf{x}[n]$ belongs to "Yes", and $y_{1}[n]<y_{2}[n]$ when $\mathbf{x}[n]$ belongs to "No".

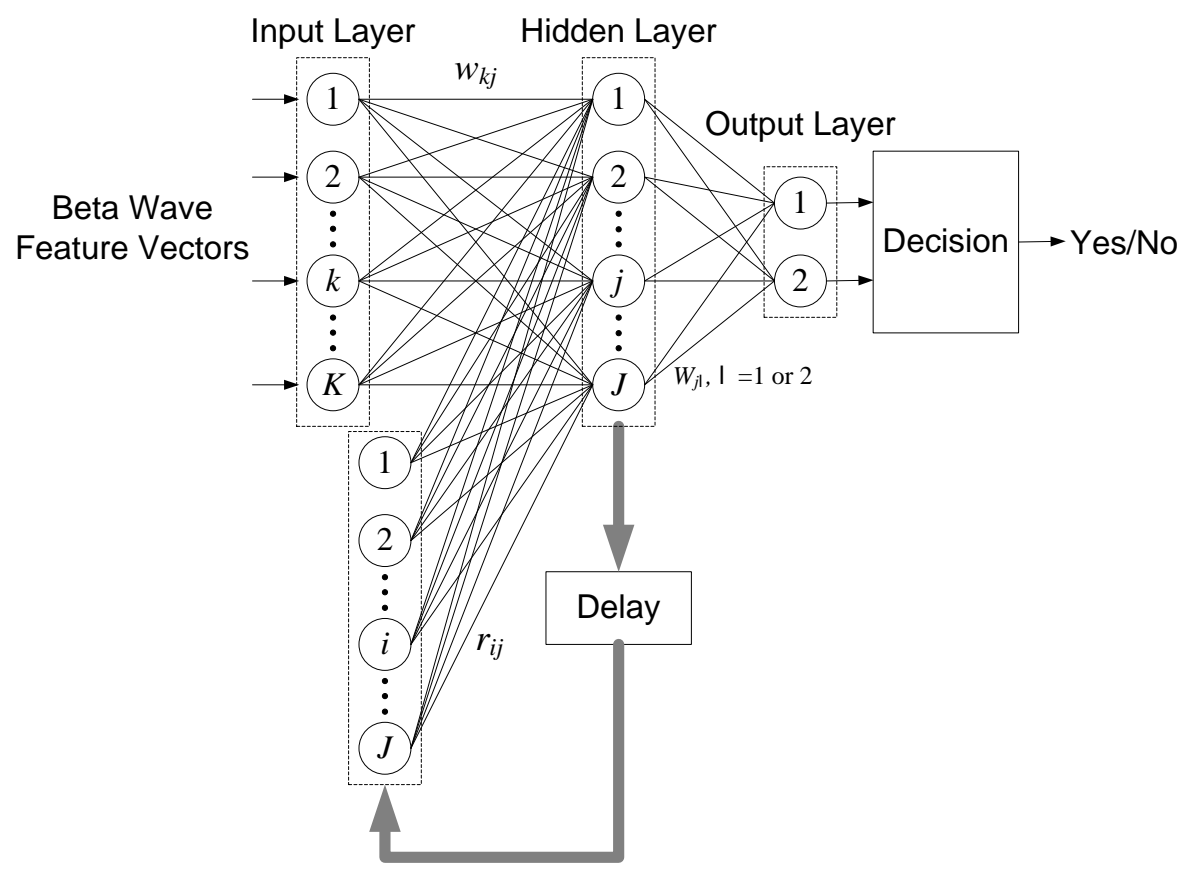

Figure. 4. Structure of our RNN.

In the testing phase, the RNN takes as input the beta wave feature vectors, and produces as output the decision on "Yes" or "No" using 


$$
\sum_{n=1}^{L} y_{1}[n]-\sum_{n=1}^{L} y_{2}[n] \stackrel{\text { Yes }}{\underset{\text { No }}{\leq}} \delta,
$$

Furthermore, we create two RNNs, one taking care of the high beta wav EEG and the other taking care of the low beta EEG. The two RNNs are then combined using a weighting strategy. Specifically, a test EEG signal is hypothesized as either "Yes" or "No" using

$$
b\left(\sum_{n=1}^{L} y_{1}^{1}[n]-\sum_{n=1}^{L} y_{2}^{1}[n]\right)+(1-b)\left(\sum_{n=1}^{L} y_{1}^{2}[n]-\sum_{n=1}^{L} y_{2}^{2}[n]\right) \underset{\text { No }}{>} \delta,
$$

where $y_{\ell}^{1}[n]$ and $y_{\ell}^{2}[n], \square=1$ and 2, are the outputs of the $\beta_{1}$-trained RNN and $\beta_{2}$-trained $\mathrm{RNN}$, respectively, $\delta$ is a pre-defined threshold, and $b$ is a weighting factor.

\section{EXPERIMENTS}

\subsection{Databases}

Our EEG data was collected from ten male subjects. Each subject was invited to undergo the EEG measurements on 6 discontinuous days, in which the data collected on the former three days were used for training, and the data collected on the latter three days were used for testing. In the testing set, we segmented the EEG recordings into 2400 test samples. Each sample is associated with a ground-truth of "Yes" or "No" when a subject saw a set of ten commands prompted on the screen. Thus, there are 240 "Yes" test samples and 2160 "No" test samples.

\subsection{Experiment Results}

Table 1 shows the results of the GMM-based command recognition, i.e., using Eq. (4), in which the accuracy is defined as

$$
\frac{\text { \#Correctly recognized Samples }}{\text { \#Testing Samples }} \times 100 \% \text {. }
$$

Note that $a=1.0$ in Eq. (4) represents using $\beta_{1}$-trained GMMs solely, and $a=0.0$ represents using $\beta_{2}$-trained GMMs solely. It can be seen from Table 1 that the best performance (in boldface letter) of the $\beta_{2}$-trained GMMs is slightly better than that of the $\beta_{1}$-trained GMMs. When combining $\beta_{1}$-trained and $\beta_{2}$-trained GMMs, i.e., $a=0.35$, the performance can be further improved. 
Table 1. Accuracy (\%) of the GMM-based command recognition, i.e., using Eq. (4).

\begin{tabular}{|c|c|c|c|}
\hline No. of Mixtures & $\begin{array}{c}\beta_{1} \text {-trained GMMs } \\
(a=1.0)\end{array}$ & $\begin{array}{c}\beta_{2} \text {-trained GMMs } \\
(a=0.0)\end{array}$ & $a=0.35$ \\
\hline 8 & 59.0 & 59.9 & - \\
\hline 16 & 60.2 & 60.8 & - \\
\hline 32 & $\mathbf{6 2 . 9}$ & $\mathbf{6 3 . 4}$ & $\mathbf{6 4 . 6}$ \\
\hline 64 & 61.9 & 61.2 & - \\
\hline
\end{tabular}

Table 2 shows the results of the HMM-based command recognition. Compared to Table 1, we can see that HMM-based recognizer is better than GMM-based recognizer in this case. When combining $\beta_{1}$-trained and $\beta_{2}$-trained HMMs, i.e., $a=0.4$, the best recognition accuracy is $78.8 \%$. On the other hand, Table 3 shows the results of the RNN-based command recognition, i.e., using Eq. (8). We can see from Tables 2 and 3 that RNN-based recognizer is slightly better than HMM-based recognizer, which achieves the best accuracy of $79.2 \%$.

Table 2. Accuracy (\%) of the HMM-based command recognition.

\begin{tabular}{|c|c|c|c|}
\hline No. of States & $\begin{array}{c}\beta_{1} \text {-trained HMMs } \\
(a=1.0)\end{array}$ & $\begin{array}{c}\beta_{2} \text {-trained HMMs } \\
(a=0.0)\end{array}$ & $a=0.4$ \\
\hline 2 & 70.4 & 68.8 & - \\
\hline 3 & $\mathbf{7 3 . 3}$ & 73.8 & - \\
\hline 4 & $\mathbf{7 3 . 3}$ & $\mathbf{7 4 . 6}$ & $\mathbf{7 8 . 8}$ \\
\hline 5 & 72.9 & 74.2 & - \\
\hline
\end{tabular}

Table 3. Accuracy (\%) of the RNN-based command recognition, i.e., using Eq. (8).

\begin{tabular}{|c|c|c|c|}
\hline $\begin{array}{c}\text { No. of Neurons in the Hidden } \\
\text { Layer }\end{array}$ & $\begin{array}{c}\beta_{1} \text {-trained RNN } \\
(b=1.0)\end{array}$ & $\begin{array}{c}\beta_{2} \text {-trained RNN } \\
(b=0.0)\end{array}$ & $b=0.6$ \\
\hline 10 & 72.9 & 72.5 & - \\
\hline 11 & 72.1 & 72.9 & - \\
\hline 12 & 72.5 & 73.3 & - \\
\hline 13 & $\mathbf{7 4 . 2}$ & 74.6 & - \\
\hline 14 & 73.8 & 74.2 & - \\
\hline 15 & 73.8 & $\mathbf{7 5 . 4}$ & $\mathbf{7 9 . 2}$ \\
\hline 16 & 73.3 & 75.0 & - \\
\hline 17 & 72.9 & 73.8 & - \\
\hline 18 & 72.9 & 74.2 & - \\
\hline 19 & 72.5 & 72.9 & - \\
\hline 20 & 71.3 & 71.7 & \\
\hline
\end{tabular}




\section{Conclusions}

This study has investigated the problem of recognizing users' commands by analysing their EEGs. In contrast to the existing studies, which rely on expensive equipment, the proposed system is rather advantageous to develop mobile-based applications, because we use a simple, portable, and cheap instrument to acquire single-channel EEGs.

Although the recognition accuracy obtained with our system leaves much room to improve, we believe that such a pilot investigation has laid a good foundation for the future development of a brain-control interface using single-channel EEGs. To be more feasible, we have to further improve the recognition accuracy. Considering that the beta wave features acquired from Mindband may not be sufficient to determine a user's command, we will study other features extracted from EEG in the future. Furthermore, we will extend the current recognition system from a user-dependent mode to a user-independent mode, so that the system can be built without using EEG training data from each user.

\section{ACKNOWLEDGEMENTS}

This work was supported in part by the National Science Council under Grants NSC101-2221E-027-128-MY2 and MOST106-2221-E-027-125-MY2.

\section{REFERENCES}

[1] L. C. Parra, C. Alvino, A. Tang, B. Pearlmutter, N. Young, A. Osman, and P. Sajda, "Linear spatial integration for single-trial detection in encephalography," Neuroimage, vol. 17, pp. 223230, 2002.

[2] L. C. Parra, C. D. Spence, A. D. Gerson, and P. Sajda, "Recipes for the Linear Analysis of EEG," Neuroimage, vol. 28, no. 2, pp. 342-353, 2005.

[3] M. G. Philiastides and P. Sajda, "Temporal characterization of the neural correlates of perceptual decision making in the human brain," Cerebral Cortex, vol. 16, pp. 509-518, 2006.

[4] M. G. Philiastides, R. Ratcliff, and P. Sajda, "Neural representation of task difficulty and decision making during perceptual categorization: a timing diagram," Journal of Neuroscience, vol. 26, pp. 8965-8975, 2006.

[5] K. Tanaka, K. Matsunaga, and H. O. Wang, "Electroencephalogram-based control of an electric wheelchair," IEEE Transactions on Robotics, vol. 21, no. 4, pp. 762-766, August 2005.

[6] B. Rebsamen, C. L. Teo, Q. Zeng, and H. Marcelo, E. Burdet, C. Guan, H. Zhang, and C. Laugier, "Controlling a wheelchair indoors using thought," IEEE Intelligent Systems, vol. 22, no. 2, pp. 18 24, 2007.

[7] K. Q. Shen, C. J. Ong, X. P. Li, Z. Hui, and P. V. Einar, "A feature selection method for multilevel mental fatigue EEG classification," IEEE Transactions on Biomedical Engineering, vol. 54, no. 7, pp. 1231-1237, July 2007.

[8] C. Zhao, C. Zheng, M. Zhao1, J. Liu, and Y. Tu, "Automatic classification of driving mental fatigue with EEG by wavelet packet energy and KPCA-SVM," International Journal of Innovative Computing, Information and Control, vol. 7, no. 3, pp. 1157-1168, 2011.

[9] J. W. Bang, E. C. Lee, and K. R. Park, "New computer interface combining gaze tracking and brainwave measurements," IEEE Transactions on Consumer Electronics, vol. 57, no. 4, pp. 16461651, November 2011.

[10] W. H. Tsai and W. B. Jheng, "Command Recognition Based on Single-Channel Electroencephalography," Journal of Information Science and Engineering, vol. 32, no. 1, pp. 229$241,2016$. 
[11] F. Sharbrough, G. E. Chatrian, R. P. Lesser, H. Luders, M. Nuwer, and T. W. Picton "American electroencephalographic society guidelines for standard electrode position nomenclature," Journal of Clinical Neurophysiology, vol. 8, pp. 200-202, 1991.

[12] Y. Yasui, "A brainwave signal measurement and data processing technique for daily life applications," Journal of Physiological Anthropology, vol. 28, no. 3, pp. 145-150, 2009.

[13] G. Rebolledo-Mendez, S. Freitas, J. R. Rojano-Caceres, A. R. Garcia-Gaona1, "An empirical examination of the relation between attention and motivation in computer-based education: a modelling approach," in Proceedings of the Twenty-Third International Florida Artificial Intelligence Research Society Conference, 2010.

[14] L. R. Rabiner. "A tutorial on Hidden Markov Models and selected applications in speech recognition," Proceedings of the IEEE, vol. 77, no. 2, pp. 257-286, 1989.

[15] A. Dempster, N. Laird, and D. Rubin, "Maximum likelihood from incomplete data via the EM algorithm," J. R. Statist. Soc., vol. 39, pp. 1-38, 1977.

[16] A. M. Ouksel, "Big data: Some research challenges in future information systems," International Journal on Information Systems and Technologies (IJIST), vol. 1, no. 1, 2013.

\section{Authors}

Wei-Ho Tsai received his M.S. and Ph.D. degrees in Communication Engineering from National Chiao-Tung University, Hsinchu, Taiwan, in 1997 and 2001, respectively. He is currently a Professor in the Department of Electronic Engineering \& Graduate Institute of Computer and Communication Engineering, National Taipei University of Technology, Taiwan. His research interests include spoken language processing and music information retrieval. Dr. Tsai is a life member of ACLCLP and a member of IEEE.

Cin-Hao Ma received the B.S. degree in electronic engineering from National Taipei University of Technology, Taipei, Taiwan, in 2012. He is pursuing the Ph.D. degree in computer and communication engineering at National Taipei University of Technology currently. His research interests include signal processing and multimedia applications

Varinya Phanichraksaphong received her B.S. degrees in Electronic Engineering from King Mongkut's Institute of Technology Ladkrabang, Bangkok, Thailand. She is currently pursuing her M.S. degrees in the Department of Electrical Engineering and Computer Science (EECS) National Taipei University of Technology, Taipei, Taiwan. Her research interests include musical instruments sound analysis and computer music processing

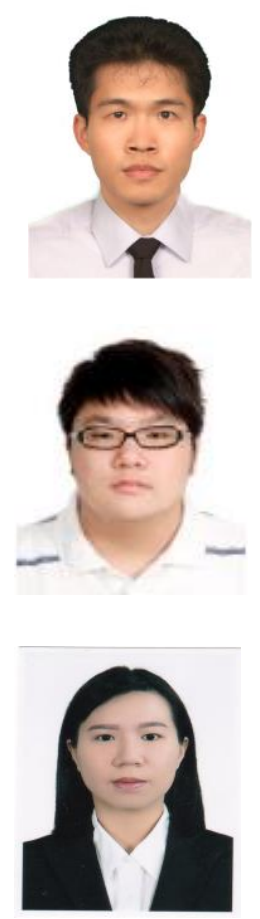

